\title{
Aplicação de Realidade Aumentada Móvel para Apoio à Alfabetização de Crianças com Autismo
}

\author{
Flávia Gonçalves Fernandes ${ }^{1}$, Luciene Chagas de Oliveira ${ }^{1}$, \\ Eduardo Chagas de Oliveira ${ }^{1}$ \\ ${ }^{1}$ Faculdade de Engenharia Elétrica - Universidade Federal de Uberlândia (UFU) \\ Uberlândia, MG - Brasil \\ flavia.fernandes92@gmail.com, lchagasoliveira@gmail.com, \\ edu_ufueyahoo.com.br
}

\begin{abstract}
Initially, it is known that education is crucial for human development. Therefore, researchers are increasingly seeking more advanced technological innovations in order to improve the quality of teaching and learning of the population. Thus, the application of Augmented Reality (AR) in education has been evaluated intensively in recent years and been highlighted, as it represents new challenges and opportunities, with the inclusion of additional information and / or relevant to the actual scenario. This work presents an application whose goal is to develop a system to support the literacy of children with autism. In this way, we hope to contribute in motivating students through technological innovations, creating a pleasant and harmonious learning environment, and effectiveness in children's learning.
\end{abstract}

Resumo. Inicialmente, sabe-se que a educação é crucial para o desenvolvimento humano. Por isso, pesquisadores buscam inovações tecnológicas cada vez mais avançadas a fim de melhorar a qualidade de ensino e aprendizagem da população. Desse modo, a aplicação da Realidade Aumentada (RA) na área da educação tem sido avaliada de forma intensiva nos últimos anos e merecido destaque, pois representa novos desafios e potencialidades, com a inserção de informações complementares elou relevantes ao cenário real. Assim, este trabalho apresenta uma aplicação cujo objetivo é o desenvolvimento de um sistema para apoio à alfabetização de crianças com autismo. Desta forma, espera-se contribuir na motivação de alunos, através das inovações tecnológicas, gerando um ambiente educacional agradável e harmonioso, além da eficácia no aprendizado infantil.

\section{Introdução}

O autismo é um transtorno global do desenvolvimento que aparece nos três primeiros anos de vida. Além disso, afeta o desenvolvimento normal do cérebro relacionado às habilidades sociais e de comunicação. Ele é marcado por três características fundamentais: inabilidade para interagir socialmente; dificuldade no domínio da linguagem para comunicar-se ou lidar com jogos simbólicos; e padrão de comportamento restritivo e repetitivo [Campanário, 2008]. 
Aplicações da tecnologia para auxiliar pessoas que possuem autismo são muito importantes. A Realidade Aumentada (RA) é uma tecnologia nova que pode ser utilizada para aplicações em diversas áreas e em constante crescimento, sendo uma subárea da realidade virtual. Também é uma tecnologia dependente de processamento em tempo real e, por isso, é influenciada pela evolução da computação, tanto do ponto de vista do hardware quanto do software [Cardoso, Lamounier e Tori, 2001].

O desenvolvimento de aplicações de Realidade Aumentada utilizando dispositivos móveis proporciona um ambiente virtual tridimensional mais atrativo e interessante para facilitar as tarefas do dia-a-dia, auxiliar diagnósticos e tratamentos de doenças, além de facilitar o aprendizado e comunicação de crianças e adultos [Lamounier e Cardoso, 2004].

A partir desse conceito, dentre várias opções na área tecnológica, pode-se citar que a Realidade Aumentada (RA) tem sido bastante utilizada para a construção do conhecimento, tornando prática e dinâmica a interatividade do usuário juntamente com a participação da mobilidade oferecida pelos dispositivos móveis, tais como, smartphones e tablets [Lecheta, 2010].

Alguns fatores de mercado contribuem para efetivação de aplicativos e softwares com a RA, como a diminuição do custo dos equipamentos tecnológicos adequados para o desenvolvimento e utilização desses aplicativos, que se tornam cada vez mais acessíveis nas diversas classes sociais.

Além disso, em virtude da amplitude em que a RA vem atuando, a sua utilização se torna imprescindível e coerente para várias soluções, contribuindo para $\mathrm{o}$ desenvolvimento social e tecnológico.

Isoladamente, as inovações tecnológicas não atingem os objetivos esperados da complexa tarefa de ensino e aprendizagem. Porém, podem ser vistos como ferramentas de apoio didático ou mesmo, meios estratégicos, empregados em diversos momentos para auxiliar pessoas com algum tipo de deficiência física. Nesta linha de raciocínio, este trabalho tem como objetivo propor uma nova ferramenta de RA para dispositivos móveis que auxilia na alfabetização de pessoas com autismo.

Com dificuldades de interagir socialmente e de se comunicar, e um padrão restrito e repetitivo de comportamento, as crianças com autismo muitas vezes se fecham em um mundo particular. Logo, esta aplicação pode ser utilizada como um recurso pedagógico para suporte e inclusão de crianças autistas, uma vez que motiva o aprendizado de maneira interativa, o que pode tornar o processo de alfabetização mais prazeroso e eficaz.

\section{Realidade Aumentada Móvel}

Alguns aparelhos de telefone celular são dispositivos que apresentam um bom poder de processamento, levando-se em conta que se tratam de dispositivos móveis microprocessados com características limitadas. Estes são capazes de executar algoritmos desenvolvidos nas linguagens $\mathrm{C}++$ e Java.

A versão Java Micro Edition (ME) define um ambiente flexível para a construção de aplicativos destinados a dispositivos móveis, como celulares, PDAs, TV set-top boxes e impressoras. O Java ME inclui interface com o usuário, segurança robusta e protocolos de comunicação em rede [Lecheta, 2010]. Um conjunto mínimo de 
características do Java ME deve ser suportado pelo dispositivo para este estar apto a rodar uma aplicação de RA, como: Mobile Information Device Profile 2.0, Mobile Media API, Connected Limited Device Configuration 1.1.

Um exemplo do uso de RA com dispositivos móveis de fácil acessibilidade é o Digital Scrap Book, onde as anotações de um álbum de recordações físico servem como marcadores para acessar conteúdos multimídia como vídeos, fotos e áudios [Milgram, 1994]. Ao posicionar a câmera do celular na frente do álbum o marcador é reconhecido pelo software e o conteúdo multimídia relacionado é apresentado pelo celular.

Outro exemplo de uso da RA é o sistema de museu virtual [Kirner e Zorzal, 2005] onde marcadores são posicionados nas paredes do museu, próximo às obras. $O$ aparelho celular, ou outro dispositivo móvel, ao capturar a imagem do marcador, está apto a mostrar na tela do aparelho, ou vocalmente, informações adicionais sobre a obra exposta.

Os exemplos citados falam de aplicativos que precisam estar conectados a uma rede externa através de tecnologia Bluetooth ou outro tipo de rede sem fio para obter dados adicionais. Nem todas as soluções precisam estar conectadas a uma rede externa. Um exemplo é um jogo de futebol para celular, onde uma bola virtual na tela do aparelho pode ser chutada com o próprio pé do usuário simplesmente apontando a câmera para o chão. A posição do pé é detectada em relação à bola virtual [Kirner e Zorzal, 2005].

\section{Trabalhos Relacionados}

Nesta seção serão descritos alguns trabalhos relacionados ao ambiente virtual e sua contribuição na área da educação.

O sistema AR Chemical Connection apresenta em sua interface um painel lateral que mostra a descrição das ligações químicas, facilitando a visualização das informações expostas. A Realidade Virtual e a Realidade Aumentada podem contribuir e melhorar representações simulando fenômenos como se de fato existissem naquele exato momento, proporcionando a visualização e interação do estudante com $o$ conhecimento de modelos químicos de maneira completa, facilitando a compreensão dos conceitos químicos, ou para correção de concepções inadequadas de modelos científicos. Nesta aplicação, o usuário então coloca dois marcadores na área de captura de imagem da câmera. Em seguida o sistema, através do FlartoolKit, faz a detecção e o rastreamento dos marcadores e projeta os objetos tridimensionais sobre eles, conforme pode ser observado na Figura 1 [Araújo, 2009].

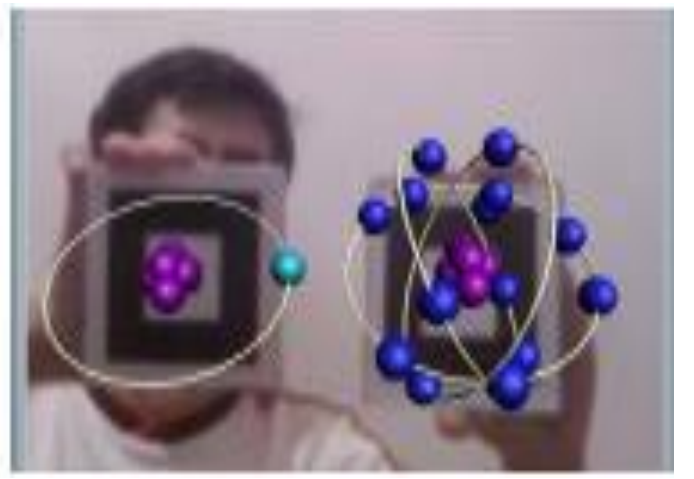

Figura 1: Objetos virtuais projetados sobre os marcadores [Araújo, 2009]. 
V Congresso Brasileiro de Informática na Educação (CBIE 2016)

Anais dos Workshops do V Congresso Brasileiro de Informática na Educação (CBIE 2016)

Um exemplo de aplicação existente que utiliza interação natural é a Fusion4D do projeto VIDA [Woodill, 2010], ilustrado na Figura 2, é um trabalho que foi desenvolvido uma aplicação 3D na qual o usuário interage com os objetos virtuais como se estivessem realmente em suas mãos, podendo mover, girar, aumentar, explodir em detalhes e até mesmo ver como os objetos seriam no passado e no futuro.

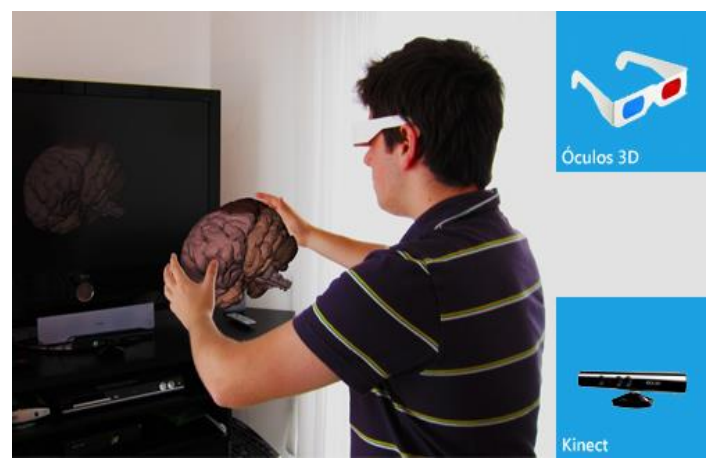

Figura 2: Projeto Fusion4D [Woodill, 2010].

Também existe um Sistema de Percepção 3D para Deficientes Visuais (SP3DDV) com o intuito de auxiliar as Pessoas com Necessidades Especiais Visuais (PNEV's) a apreensão de conceitos até então desconhecidos através da realidade aumentada (Figura 3). Além disso, O SDP3D-DV pode tornar acessível aos deficientes visuais a apreciação das artes pictóricas, em museus e outras instituições similares, através de uma nova rede de conceitos com o sistema tátil, onde o imaginário e a memória visual buscam o reconhecimento para apreciação dos referidos artefatos artístico [KukulskaHulme e Traxler, 2005].

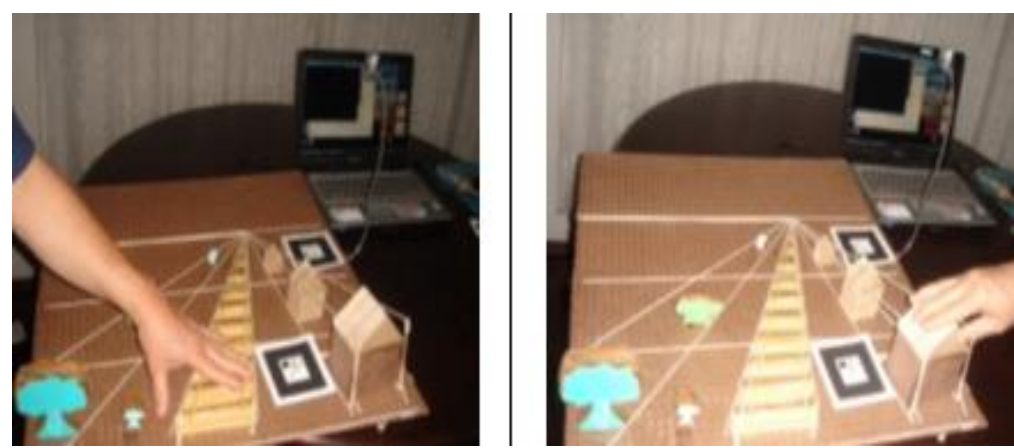

Figura 3: SP3D-DV em funcionamento [Kukulska-Hulme eTraxler, 2005].

Além disso, há um Sistema Interativo com Realidade Aumentada para Ensino da Anatomia (SIRAEA), onde é representada, como na Figura 4, a estrutura de um crânio tridimensional (3D) e permite aos usuários desmontar, montar e manipular o crânio 3D de forma intuitiva utilizando apenas webcam e marcador [Tori et al, 2006]. 
V Congresso Brasileiro de Informática na Educação (CBIE 2016)

Anais dos Workshops do V Congresso Brasileiro de Informática na Educação (CBIE 2016)

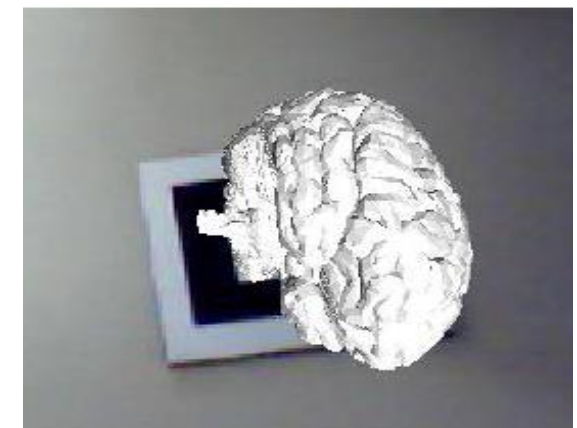

Figura 4: Cérebro virtual em Realidade Aumentada [Tori et al, 2006].

Também já foi implementada uma Aplicação Educacional aplicada à Medicina utilizando Realidade Aumentada (AEMRA), em que o seu desenvolvimento é realizado com uso da biblioteca ARToolKit. Nesse trabalho, há exemplos de sobreposição de órgãos sobre os marcadores, como o coração virtual em RA da Figura 5, que permite visualização e manipulação do respectivo órgão com aspectos diferenciados tornando o aprendizado mais dinâmico e realista [Costa e Ribeiro, 2009].

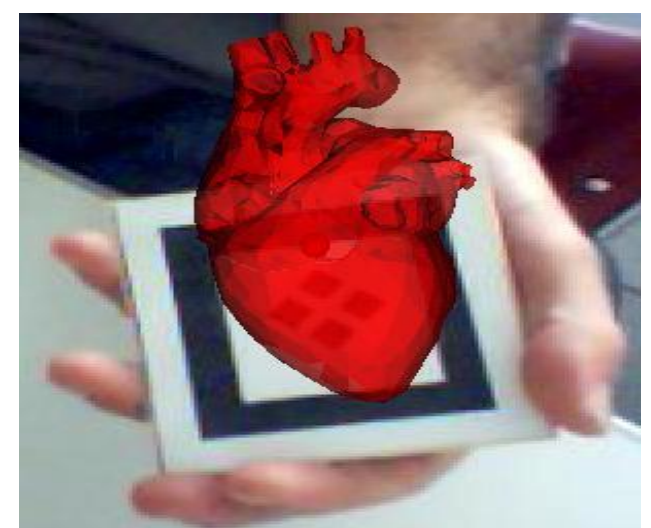

Figura 5: Coração virtual em Realidade Aumentada [Costa e Ribeiro, 2009].

E, ainda, foi desenvolvido um Ambiente de Realidade Aumentada para ensinoaprendizagem de Diabetes Mellitus (ARADM), que foi realizado por meio de representações tridimensionais, interativas e em tempo real, de seus principais aspectos anatômicos e fisiopatológicos, como pode ser visto na Figura 6 [Drab e Artner, 2005].

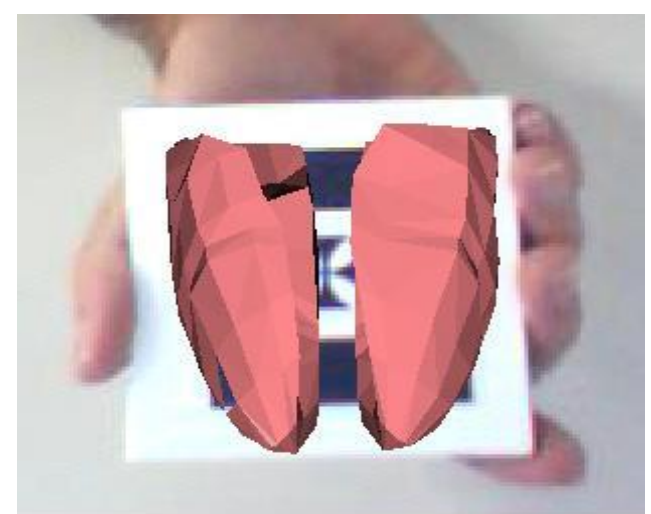

Figura 6: Rins virtuais em Realidade Aumentada [Drab e Artner, 2005]. 
V Congresso Brasileiro de Informática na Educação (CBIE 2016)

Anais dos Workshops do V Congresso Brasileiro de Informática na Educação (CBIE 2016)

\section{Aplicação de RA Móvel para Alfabetização de Crianças Autistas}

Neste trabalho foi desenvolvida uma aplicação para auxílio na alfabetização de crianças autistas utilizando RA para dispositivos móveis.

Esse sistema tem como objetivo ajudar crianças com dificuldades no aprendizado escolar e, principalmente, na alfabetização, relacionando-as com imagens que são apresentadas no mundo real, no formato tridimensionais, utilizando a tecnologia da RA móvel.

Essa didática visa conhecimento básico do mundo ao redor da criança, em que a interação entre mundo real e virtual estará disponível em um serviço móvel tonando mais dinâmico o processo de aprendizagem da criança.

Nesta aplicação, a criança poderá ter acesso em seu próprio dispositivo móvel adequado para a aplicação da RA, que exibirá as ilustrações como se fossem reais.

Em relação aos aspectos metodológicos e tecnológicos da implementação do sistema, foi utilizada RA por meio do desenvolvimento de ambientes virtuais, incluindo interações e animações, com uso das linguagens de programação Java e Javascript, a linguagem de marcação HTML (HyperText Markup Language) para estruturação dos conteúdos, a linguagem CSS (Cascading Style Sheets) para formatação dos conteúdos estruturados.

A aplicação foi desenvolvida no ambiente Eclipse, conectado ao emulador do sistema Android, e também utilizou-se a ferramenta de modelagem gráfica Blender, a fim de contemplar cálculos e comandos, visando criar para o usuário a possibilidade de interagir com ambientes virtuais atrativos, que facilitem a aprendizagem significativa de conceitos teóricos e práticos. Também utiliza o sistema Android para dispositivos móveis na realização de testes e para sua utilização prática e efetiva [Harma, 2003].

O emulador Android, também conhecido como Android Virtual Device (AVD), realiza o reconhecimento de características de smartphones usando a versão do Android $^{\mathrm{TM}}$ escolhida (seja a 2.1, 2.2 ou qualquer outra). Com ele, são carregados a imagem do sistema e características, onde o usuário pode realizar testes no projeto [Lecheta, 2010].

O Android SDK é um aplicativo instalado no software Eclipse que permite que os desenvolvedores elaborem as aplicações a partir de um dispositivo virtual para os aparelhos de celular e tablet, desde jogos a utilitários que façam uso das funções oferecidas pelos aparelhos, como touchscreen, telefonia GSM, Câmera, GPS, bússola, acelerômetro, Bluetooth, EDGE, 3G e WiFi. A plataforma apresenta suporte para mídias de áudio, vídeo e imagem, nos formatos MPEG4, H.264, MP3, AAC, AMR, JPG, PNG, GIF, bem como acelerador gráfico 3D, baseados no OpenGL ES. Os dados podem ser armazenados em SQLite e a plataforma traz um navegador integrado com base no código livre do motor WebKit [Neto, 2006].

Além disso, foi utilizada a biblioteca ARToolKit para a implementação desta aplicação. Ela é open source e viabiliza o desenvolvimento de interfaces para realidade aumentada. Esta ferramenta emprega métodos de visão computacional para detectar tags na imagem capturada por uma câmera. $O$ rastreamento óptico desta tag possibilita $O$ ajuste de posição e orientação para realizar a renderização de um objeto virtual, de 
V Congresso Brasileiro de Informática na Educação (CBIE 2016)

Anais dos Workshops do V Congresso Brasileiro de Informática na Educação (CBIE 2016)

modo que esse objeto pareça estar junto a tag, desta forma o usuário pode manipular o objeto virtual, utilizando um objeto real [Azuma, 2011].

Ao instalar o aplicativo em um celular que tenha sistema operacional Android, o usuário se deparará com uma tela inicial, ilustrada na Figura 7. Nela, encontra-se o menu de opções do sistema implementado, composto por Letras, Sílabas, Palavras, Números, Animais e Frutas. Após selecionar alguma dessas opções, é exibida uma lista das próximas opções disponíveis para cada um destes itens. E, os próximos itens, ao serem selecionados, são exibidos em Realidade Aumentada.

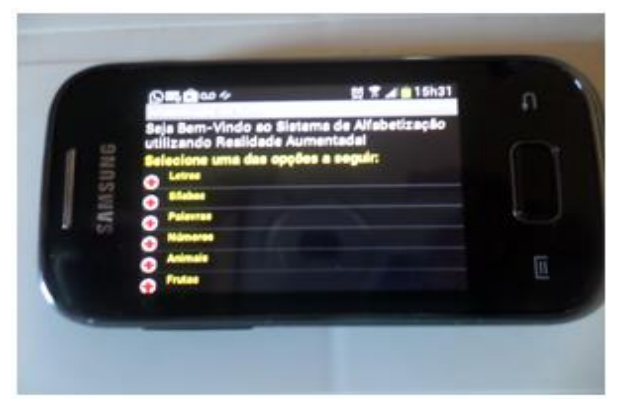

Figura 7: Tela inicial no dispositivo móvel.

O usuário após selecionar uma opção desejada, o sistema exibe a imagem 3D em um marcador para facilitar a visualização da necessidade de aprendizado da criança.

Primeiramente, espera-se ensinar as letras para as crianças. Desta forma, ao selecionar esta opção no sistema, serão exibidas todas as letras do alfabeto e, ao clicar sobre elas, as mesmas serão exibidas em RA, o que facilita a memorização da forma da letra escolhida.

As Figuras 8(a) e 8(b) exibem as letras A e T, respectivamente, em Realidade Aumentada por meio do dispositivo móvel sobre o marcador.

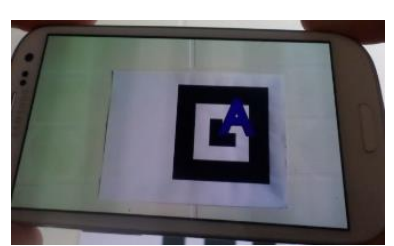

(a)

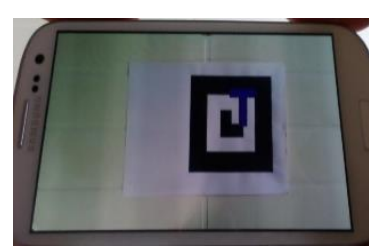

(b)

Figura 8: (a) Letra A exibida em RA no celular.

(b) Letra T exibida em RA no celular.

O mesmo procedimento ocorre, consequentemente, para as sílabas, conforme Figura 9(a), e as palavras, de acordo com a Figura 9(b).

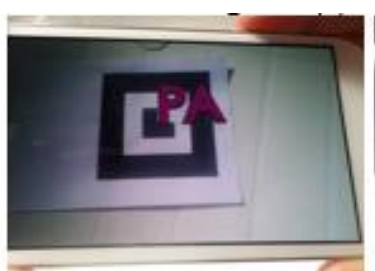

(a)

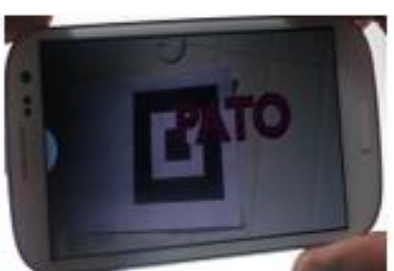

(b)

Figura 9: (a) Sílaba PA exibida em RA no celular. (b) Palavra PATO exibida em RA no celular. 
V Congresso Brasileiro de Informática na Educação (CBIE 2016)

Anais dos Workshops do V Congresso Brasileiro de Informática na Educação (CBIE 2016)

A seção Números contém a lista de algarismos de 0 a 9 em RA. E, posteriormente, os números formados por estes algarismos, mostrando a contagem de 0 a 100 em Realidade Aumentada.

As Figuras 10(a) e 10(b) exibem os números 4 e 8 , respectivamente, em Realidade Aumentada por meio do dispositivo móvel sobre o marcador.

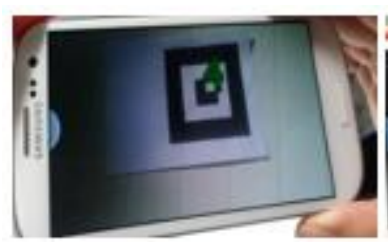

(a)

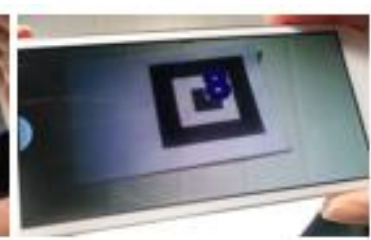

(b)

Figura 10: (a) Número 4 exibido em RA no celular. (b) Número 8 exibido em RA no celular.

$\mathrm{Na}$ seção Animais, há uma lista com os principais animais existentes, e os mesmos podem ser exibidos em RA ao clicar sobre eles. O mesmo acontece na seção Frutas.

As Figuras 11(a) e 11(b) exibem animais em Realidade Aumentada por meio do dispositivo móvel sobre o marcador: uma vaca e um pato, respectivamente.

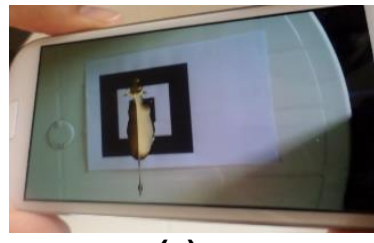

(a)

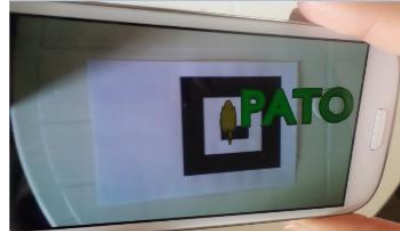

(b)

Figura 11: (a) Vaca exibida em RA no celular. (b) Pato exibido em RA no celular.

As Figuras 12(a) e 12(b) exibem o animal cão e a fruta maçã, respectivamente, em Realidade Aumentada por meio do dispositivo móvel sobre o marcador.

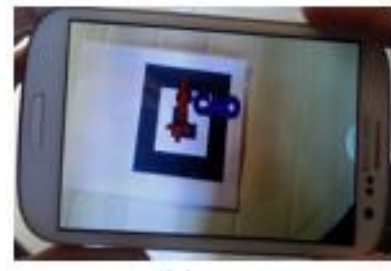

(a)

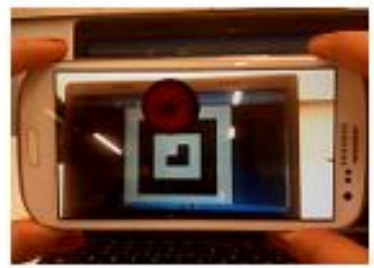

(b)

Figura 12: (a) Cão exibido em RA no celular.

(b) Maçã exibida em RA no celular.

A plataforma Android atualmente é tecnologia predominante nos dispositivos móveis e de baixo custo que vem ganhando grande espaço no mercado tecnológico. Também compõem este aplicativo as imagens tridimensionais criadas e desenvolvidas na plataforma Blender que dispõe de uma variedade ferramentas para manipular e criar imagens em 3D com contornos e perfeitos [Blender, 2015]. 
Para o desenvolvimento da ferramenta foi utilizado o conceito da visão pelo vídeo ou tela do dispositivo móvel através da captura da imagem a partir da câmera do aparelho apontada no marcador. O marcador é predefinido no sistema que reconhece as imagens retornando o resultado a partir da escolha realizada pelo usuário no menu inicial do aplicativo.

Após a conclusão de desenvolvimento do aplicativo, pode-se analisar as necessidades de melhoramento para trabalhos futuros, além disso, pode-se destacar já a importância da utilidade do aplicativo no desenvolvimento da comunicação de crianças que têm deficiência na fala.

Diante dos modelos desenvolvidos, a ferramenta possui imagens do cotidiano, a assimilação da imagem e a palavra é a combinação perfeita para o entendimento do usuário.

O sistema consegue reconhecer o marcador a partir de qualquer superfície plana ou não com ambiente de luminosidade clara ou natural.

\section{Conclusões}

Em virtude do que foi mencionado, observa-se que há uma crescente expansão da Realidade Aumentada na implementação de ferramentas e sua contribuição no desenvolvimento de aplicativos na área da educação, saúde e medicina.

A medicina é uma das áreas de grande demanda ao uso de RA em educação, considerando treinamento, diagnóstico, tratamento e simulação de cirurgias. Pelas suas características de visualização 3D e de interação em tempo real, permite a realização de aplicações médicas inovadoras, que antes não podiam ser realizadas.

A RA é uma área tipicamente multidisciplinar que envolve conceitos provenientes de diversos segmentos, com aplicação em várias áreas ou campos específicos da vida econômica, social e cultural. Por permitir uma interação humanocomputador mais natural em ambiente tridimensional (3D) e possibilitar a reprodução de situações reais, torna-se um recurso de amplo potencial.

Os dispositivos móveis são veículos essenciais para o uso de várias tecnologias e todas as suas funções atuais tenderão a ficar obsoletas, provocando assim a evolução no sentido do multifuncional, ou seja, o "mobile" passará a ser "all mobile", composto por um número de serviços e aplicações multimídia, unindo o streaming de áudio e vídeo, o acesso à Internet, informação georreferenciada, informação em tempo real, realidade aumentada, entre outros, enquanto houver limites para transpor na nossa imaginação.

A partir de suas características de usabilidade, dinamismo, capacidade de promover a interação entre os mundos real e virtual, torna a capacidade de comunicar diante da disposição de se ter o conhecimento no cotidiano em qualquer lugar.

Com isso, este trabalho apresentou a utilização da tecnologia de Realidade Aumentada combinada a aprendizagem móvel para criação de ambientes de auxílio à alfabetização, permitindo ampliar as possibilidades de aprendizado de crianças com autismo.

Como proposta de melhorias futuras, propõe-se adicionar novas funcionalidades na aplicação, tais como, a busca dos itens cadastrados e a escolha de outras imagens, bem como realizar uma avaliação de resultados com crianças autistas. 
V Congresso Brasileiro de Informática na Educação (CBIE 2016)

Anais dos Workshops do V Congresso Brasileiro de Informática na Educação (CBIE 2016)

\section{Referências}

Araújo, D. M. et al. Uso de realidade aumentada como ferramenta complementar ao ensino das principais ligações entre átomos. In: VI Workshop de Realidade Virtual e Aumentada, Universidade de Santa Cecília, 2009.

Azuma, R.; Billinghurst, M. and Klinker, G.. 2011. "Editorial: Special Section on Mobile Augmented Reality", in Computer Graphics. pp. vii-viii.

Blender, 2015, [Online; accessed 20-Set-2012]. [Online]. Available: http://www.blender.org/.

Campanário, I. S. Espelho, espelho meu: A psicanálise e o tratamento precoce do autismo e outras psicopatologias graves. Salvador: Ágalma, 2008.

Cardoso, A. ; Lamounier Jr. and Tori, R. (2001), “Interactive 3D Physics Experiments through the Internet", Proceedings of the 4th SBC Symposium on Virtual Reality, pp.280-90, Florianópolis, Santa Catarina, Brazil. http://users.hub.ofthe.net/ mtalkmit/veshtml2.html, November.

Costa, R. M.; Ribeiro, M. W. Aplicações de realidade virtual e aumentada. Porto Alegre: SBC, 2009. 146 p.

Drab, S.; Artner, N M. Motion Detection as Interaction Technique for Games \& Applications on Mobile Devices. Pervasive Mobile Interaction Devices, Munich, Alemanha, 2005.

Harma, A. et al. Techniques and applications of wearable augmented reality audio. In: Audio Engineering Society Convention Paper, Amsterdam, Holanda, 2003.

Kirner, C; Zorzal, E.'Jogos Educacionais em Ambiente de Realidade Aumentada". Workshop de Realidade Aumentada, Piracícaba- São Paulo, 2005.

Kukulska-Hulme, A., and Traxler, J. (eds.) (2005). "Mobile Learning: A Handbook for Educators and Trainers". London: Routledge.

Lamounier, E. e Cardoso, A. Realidade virtual: uma abordagem prática. São Paulo: Mania de Livro, 2004. 326 p.

Lecheta, R. R.. Google Android, 2nd ed., Novatec, Ed., 2010. [Online]. Available: http://www.livroandroid.com.br/

Milgram, P. et. al. (1994) "Augmented Reality: A Class of Displays on the RealityVirtuality Continuum". Telemanipulator and Telepresence Technologies, SPIE, V.2351, p. 282-292.

Neto, S. et al. Criação de Aplicações de Realidade Aumentada em Dispositivos Móveis Baseados em Symbian OS. In: II Workshop de Aplicações de Realidade Virtual, Universidade Federal de Pernambuco, 2006, p. 16-19.

Tori, R.; Kirner, C.; Siscouto, R.."Fundamentos e Tecnologia de Realidade Virtual e Aumentada". Belém: VIII Symposium On Virtual Reality, 2006.

Woodill, G. "The Mobile Learning Edge: Tools and Technologies for Developing Your Teams", 1st ed., McGraw-Hill, Ed., 2010. [Online]. Available: http://www.mobilelearningedge.com 